\title{
Highly Anisotropic Dirac Fermions in Square Graphynes
}

\author{
L. Z. Zhang, ${ }^{\dagger, \neq, \S}$ Z. F. Wang, ${ }^{\S, \perp}$ Zhiming M. Wang, ${ }^{* \dagger}$ S. X. Du, ${ }^{*, \neq}$ H.-J. Gao, ${ }^{\ddagger}$ and Feng Liu ${ }^{*, \S, \|}$
}

${ }^{\dagger}$ Institute of Fundamental and Frontier Sciences, University of Electronic Science and Technology of China, Chengdu 610054, China

${ }^{\ddagger}$ Institute of Physics, Chinese Academy of Sciences, Beijing 100190, China

${ }^{\S}$ Department of Materials Science and Engineering, University of Utah, Salt Lake City, Utah 84112, United States

"Collaborative Innovation Center of Quantum Matter, Beijing 100871, China

${ }^{\perp}$ Hefei National Laboratory for Physical Science at the Microscale, University of Science and Technology of China, Hefei, Anhui 230026, China

\section{Supporting Information}

ABSTRACT: We predict a family of $2 \mathrm{D}$ carbon (C) allotropes, square graphynes (S-graphynes) that exhibit highly anisotropic Dirac fermions, using first-principle calculations within density functional theory. They have a square unit-cell containing two sizes of square $\mathrm{C}$ rings. The equal-energy contour of their 3D band structure shows a crescent shape, and the Dirac crescent has varying Fermi velocities from $0.6 \times 10^{5}$ to $7.2 \times 10^{5} \mathrm{~m} / \mathrm{s}$ along different $k$ directions. Near the Fermi level, the Dirac crescent can be nicely expressed by an extended 2D Dirac model Hamiltonian. Furthermore, tightbinding band fitting reveals that the Dirac crescent originates from the next-nearest-neighbor interactions between $\mathrm{C}$ atoms. S-graphynes may be used to build new $2 \mathrm{D}$ electronic devices taking advantages of their highly directional charge transport.

Since the discovery of graphene, ${ }^{1-3} 2 \mathrm{D}$ materials have attracted more and more attention. Especially the 2D Dirac materials, ${ }^{4-9}$ exhibiting ballistic charge transport, enormously high carrier mobility, and topological phases, ${ }^{10-12}$ offer potential applications for high performance electronic and spintronic devices. It has been also shown that highly anisotropic charge transport can be beneficial to graphene-based $2 \mathrm{D}$ devices. ${ }^{13,14}$ The highly anisotropic band dispersion around Fermi surface was also predicted in the $\mathrm{VO}_{2}-\mathrm{TiO}_{2}$ nanoheterostructures, ${ }^{15,16}$ characterized with a semi-Dirac band. Currently, there have been continued efforts in search of $2 \mathrm{D}$ materials including those with highly anisotropic Dirac states.

Among all the 2D Dirac materials, carbon (C) allotropes take up the largest portion, ${ }^{9}$ including graphene, ${ }^{1-3}$ rectangular graphenes, ${ }^{17}$ and graphynes. ${ }^{5,18-24}$ Different from graphene, graphynes contain both $\mathrm{sp}$ and $\mathrm{sp}^{2}$ hybridized $\mathrm{C}$ atoms, being the biggest $\mathrm{C}$ allotrope family. It was first proposed by Baughman et al. in 1987, ${ }^{21}$ and recent calculations show that similar to graphene, some graphynes are Dirac materials, including $\alpha$-, $\beta$-, $\delta$-, 6,6,12-, 14,14,14-, 14,14,18-, 6,6,18-, and h$12,12,20$-graphyne. ${ }^{5,19,23,24}$ On the other hand, some other $2 \mathrm{D}$ $\mathrm{C}$ structures, such as $\mathrm{S}$-graphene, $\mathrm{D}$-graphene, and E-graphene, were predicted to be Dirac materials, ${ }^{17}$ even though they have a nonhexagonal rectangular symmetry. However, it has been pointed out ${ }^{25,26}$ that no square symmetry $\mathrm{C}$ allotropes have been found to be Dirac materials.
In this work, we investigate the electronic properties of a family of square graphynes (S-graphynes) by using the firstprinciples calculations. We find that S-graphynes, containing two sizes of square $C$ rings in one unit cell, turn out to be Dirac materials and exhibit highly anisotropic Dirac crescent near the Fermi level $\left(E_{\mathrm{F}}\right)$. They are characterized by a strongly varying Fermi velocity from $0.6 \times 10^{5}$ to $7.2 \times 10^{5} \mathrm{~m} / \mathrm{s}$ along different $k$ directions. Near the Dirac point, the highly anisotropic Dirac crescent can be nicely described by an effective $2 \times 2$ Dirac Hamiltonian. Finally, by fitting the first-principles band with a tight-binding method, we reveal that the Dirac crescent originates from the next-nearest-neighbor interactions between C atoms.

All density functional theory calculations are carried by using Vienna ab initio simulation package (VASP) ${ }^{27,28}$ with the projector augmented wave method. ${ }^{29}$ Local density approximation $^{30}$ in the form of Perdew-Zunger ${ }^{31}$ is adopted for the exchange-correlation functional. The energy cutoff of the planewave basis sets is $400 \mathrm{eV}$. The Monkhorst-Pack scheme with 9 $\times 9 \times 1 k$-points is used to sample the Brillouin zone for structural optimization. To obtain an accurate density of states, $42 \times 42 \times 1 k$-points sampling is used to do the static selfconsistent calculation. In all the calculations, a $15 \AA$ vacuum

Received: June 23, 2015

Accepted: July 14, 2015 
layer is used and all atoms are fully relaxed until the residual forces on each atom are smaller than $0.001 \mathrm{eV} / \AA$. The phonon frequencies are calculated by using a supercell approach as implemented in the PHONOPY code. ${ }^{32,33}$

Figure $1 \mathrm{a}$ and $\mathrm{b}$ show the schematic structures of two kinds of S-graphynes. One $\mathrm{C}_{4}$ ring and one $\mathrm{C}_{12}$ ring in the unit cell
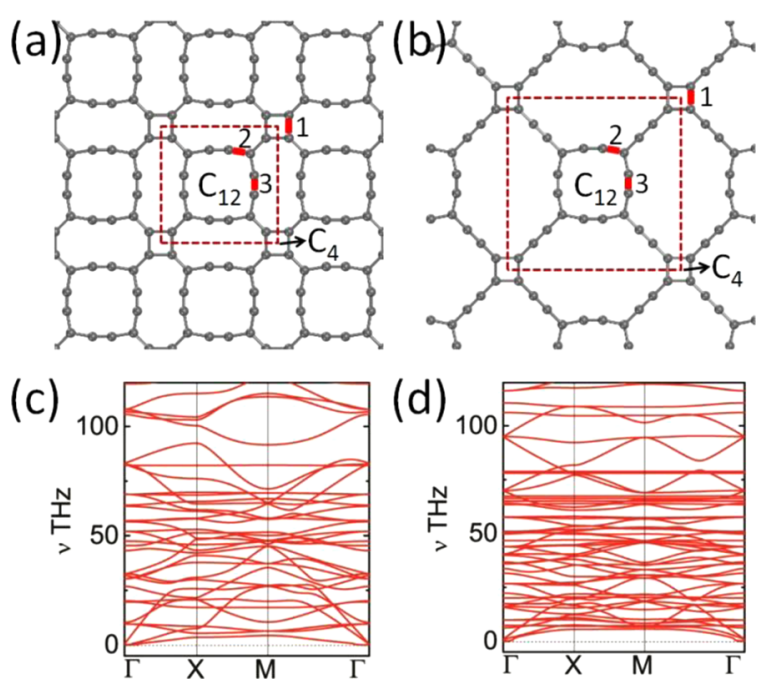

Figure 1. (Color online) Schematic structures of 4,12,2-graphyne (a) and 4,12,4-graphyne (b), the red dash squares indicate the unit cells, (c) and (d) show the phonon-dispersion curves for (a) and (b), respectively.

are indicated, which are connected with each other to form Sgraphynes. In 4,12,2-graphyne (Figure 1a), two $\mathrm{C}$ rings are connected directly; in 4,12,4-graphyne (Figure $1 \mathrm{~b}$ ), two $\mathrm{C}$ rings are connected by an acetylene bond. There are three kinds of $\mathrm{C}$ bonds, which are marked by red color. Their bond lengths are about $1.45,1.39$, and $1.22 \AA$ for 1,2 , and 3, respectively. Both S-graphynes have the plane group $p 4 m m$, with lattice constant $a_{1}=7.39 \AA$ and formation energy $E_{1}=9.03 \mathrm{eV} /$ atom for 4,12,2graphyne and $a_{2}=10.99 \AA$ and $E_{2}=8.89 \mathrm{eV} /$ atom for 4,12,4graphyne, respectively. Their formation energies are comparable with graphdiyne, which has been synthesized in experiment. ${ }^{19}$ Some large molecules containing $\mathrm{C}_{4}$ and $\mathrm{C}_{12}$ rings have been synthesized, ${ }^{34-36}$ which may be used as the building blocks for S-graphynes. We also calculated phonon-dispersion curves of these two S-graphynes, as shown in Figure 1c and d, which show the absence of imaginary frequencies.

The calculated band structures and projected density of states (PDOS) of 4,12,4-graphyne are shown in Figure 2a. The main feature is a Dirac point at B point. The two linear bands cross at the $E_{\mathrm{F}}$ and located at an off-symmetry point $\left[(0.5,0.1706) \times 2 \pi / a_{2}\right]$ between $M$ and $X$ (right inset in Figure $2 \mathrm{a}$ ). The PDOS (right panel of Figure $2 \mathrm{a}$ ) show that the Dirac states near the $E_{\mathrm{F}}$ are predominantly contributed by $\mathrm{p}_{z}$ orbital of $\mathrm{C}$. To see the band structure more clearly, we plot the $3 \mathrm{D}$ valence and conduction bands around the $E_{\mathrm{F}}$ in Figure $2 \mathrm{~b}$, which show the large anisotropy of the Dirac crescent. In the Brillouin zone, there are four equivalent Dirac points. The largest Fermi velocity along M-X direction is about $7.2 \times 10^{5}$ $\mathrm{m} / \mathrm{s}$, which is slightly smaller than that of graphene. Along other directions, the Fermi velocity is much smaller. The band structures and PDOS for 4,12,2-graphyne are very similar to those of 4,12,4-graphyne, with a Dirac points at B $[(0.5,0.1857)$ $\left.\times 2 \pi / a_{1}\right]$ (see Supporting Information Figure S1).
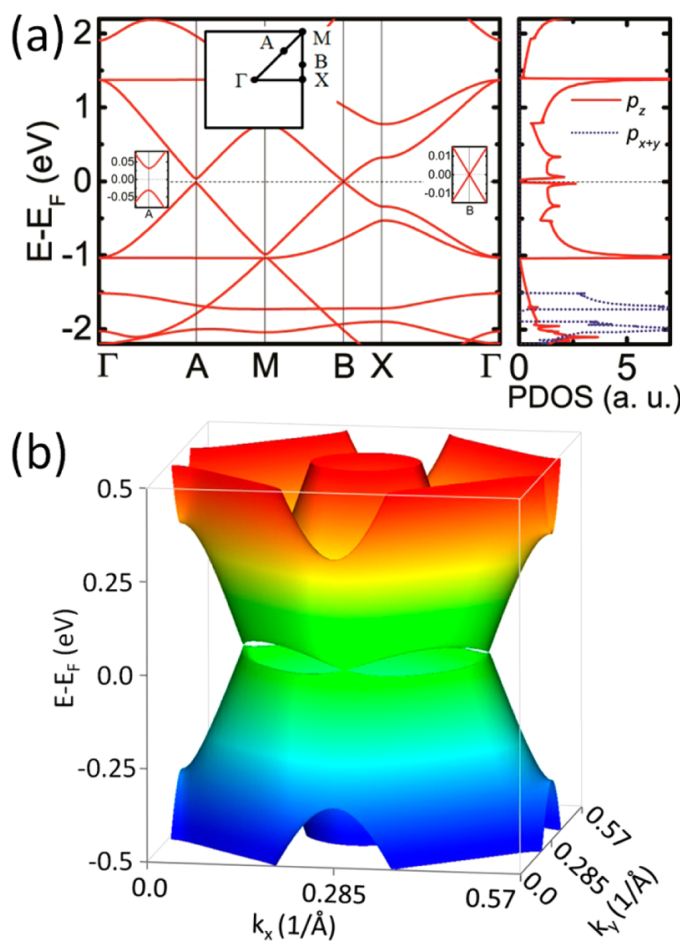

Figure 2. (Color online) Electronic structures of 4,12,4-graphyne. (a) Band structure and PDOS, where the insets indicate the first Brillouin's zone (top), the zoom-in around the A (left) and B point (right) respectively. (b) 3-D valence and conduction bands around the Fermi level for 4,12,4-graphyne.

It has been shown that acetylenic linkages in graphynes mainly mediate effective hopping between vertex $\mathrm{p}_{z}$ orbitals, which determine the existence/absence of Dirac cones in graphynes. ${ }^{17}$ Here, to describe such highly anisotropic Dirac crescent, we propose an effective $2 \times 2$ Hamiltonian $H(k)^{16,37}$

$$
H(\mathbf{k})=\mathbf{h}(\mathbf{k}) \cdot \vec{\sigma}
$$

where $\mathbf{h}(\mathbf{k})=\left(k_{x}^{2} / 2 m-v_{y} k_{y}, v_{x} k_{x}, 0\right), \vec{\sigma}=\left(\sigma_{x} \sigma_{y} \sigma_{z}\right)$. Then we have the dispersion relation of the S-graphynes as

$$
E= \pm \sqrt{\left(\frac{k_{x}^{2}}{2 m}-v_{y} k_{y}\right)^{2}+\left(v_{x} k_{x}\right)^{2}}
$$

The 3D plot of the low-energy dispersion of this model is shown in Figure 3a, which captures the highly anisotropic properties of the band structures for 4,12,4-graphyne. Figure $3 \mathrm{c}$ and $d$ show that this extended Dirac model Hamiltonian (red circles) provides a perfect description of the First-principles band structures (black line). The dispersion is linear along one direction $\left(k_{x}=0\right.$, Figure $\left.3 \mathrm{~d}\right)$ and also linear along the perpendicular direction $\left(k_{y}=0\right.$, Figure 3c) for small momentum $\left(k_{x} \sim 0\right.$, see Supporting Information Figure S2) but is parabolic for large $k_{x}$ (see Figure $3 \mathrm{c}$ ). This is slightly different from that of the semi-Dirac materials, ${ }^{9,16}$ which is linear along one direction and completely quadratic in the perpendicular direction. From the linear dispersions at the Dirac point, we obtain the effective mass and Fermi velocities to be $m=0.13 m_{e}, v_{x}=0.8 \times 10^{5} \mathrm{~m} / \mathrm{s}$ and $v_{y}=7.2 \times 10^{5} \mathrm{~m} / \mathrm{s}$ for 4,12,4-graphyne and $m=0.16 m_{e}, v_{x}=0.6 \times 10^{5} \mathrm{~m} / \mathrm{s}$ and $v_{y}=$ $5.8 \times 10^{5} \mathrm{~m} / \mathrm{s}$ for 4,12,2-graphyne (Supporting Information Figure S3). Due to the particular crescent-like band structures of S-graphyne, highly directional transport of the electron beam 

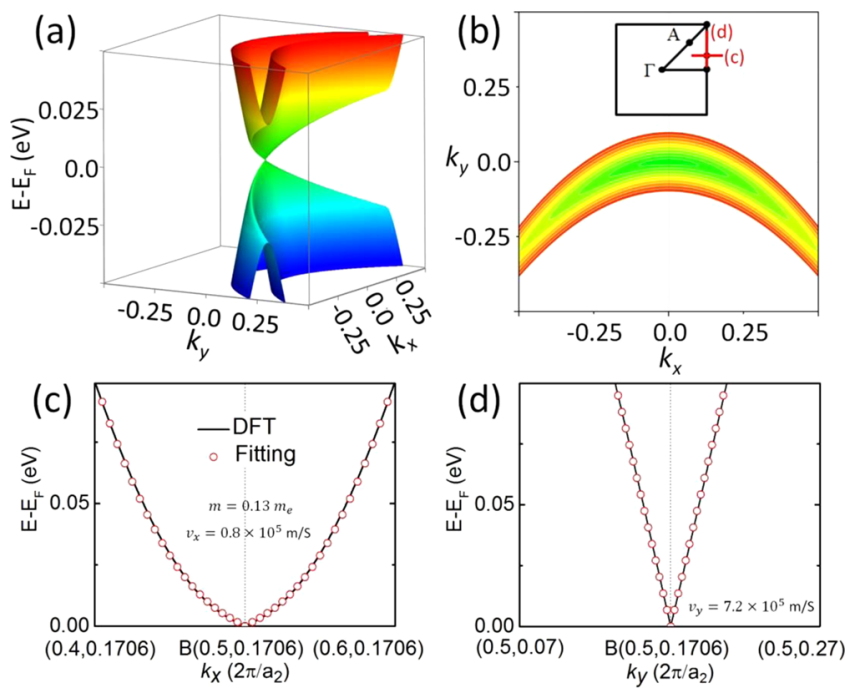

Figure 3. (Color online) 3D band structures (a) and corresponding $2 \mathrm{D}$ constant-energy contour (energy contour interval $=0.005 \mathrm{eV})(\mathrm{b})$ for the effective highly anisotropic dispersion model. (c) and (d) Firstprinciples band structures (black line) and effective model fitting results (red circle) around the Fermi level for 4,12,4-graphyne along two perpendicular directions (inset in panel b).

is expected (highly anisotropic group velocities are shown in Supporting Information Figure S4 and S5).

To understand the physical origin of such high anisotropy, we tried to fit the DFT bands structure over a large energy window by incorporating different interactions in a tightbinding (TB) model. For the electronic states around the Fermi level are predominantly contributed by $\mathrm{p}_{z}$ orbitals of $\mathrm{C}$ atom, only $\mathrm{p}_{z}$ electrons and single-orbital hoppings are included. The TB Hamiltonian could be generally written as

$$
H=\sum_{\langle i, j\rangle} t_{i, j}\left(C_{i}^{\dagger} C_{j}+h . c .\right)
$$

where the operators $C_{i}^{\dagger}$ and $C_{j}$ are the creation and annihilation operators of electrons at site $i,\langle i, j\rangle$ denotes the sum over neighbors within the unit cell, and $t_{i, j}$ stands for the hopping magnitude between sites $i$ and $j$. Considering the fact that a large number of $\mathrm{C}$ atoms are involved, to minimize the number of fitting parameters, we assume that the hopping magnitude scales with interatomic $\mathrm{C}-\mathrm{C}$ distance as

$$
t_{i, j}=t_{0} e^{c_{0}\left(1-\frac{r_{i, j}}{r_{0}}\right)}
$$

where $r_{i, j}$ stands for the distance between sites $i$ and $j, r_{0}$ is the $\mathrm{C}-\mathrm{C}$ bond length of graphene $\left(r_{0}=1.413 \AA\right)$.

Interestingly, we find that only if the next-nearest-neighbor hopping is included, we can get the accurate band structures in agreement with the first-principles results (see Supporting Information Table S1 and Figure S6). In Figure 4, we compare the $2 \mathrm{D}$ constant-energy contour of the TB model for 4,12,4graphyne. We can clearly see the Dirac crescent in Figure 4a, when the second nearest neighbor hopping is included; in contrast, no crescent but an equal-energy loop appears in Figure $4 \mathrm{~b}$, when only the nearest neighbor hopping is included.

In summary, we demonstrate existence of highly anisotropic Dirac Fermions in a class of $2 \mathrm{D}$ all-C allotropes with square symmetry. Their low-energy Fermi surface is characterized with a Dirac crescent shape. TB calculations further shows that the Dirac crescent is created by the next-nearest-neighbor

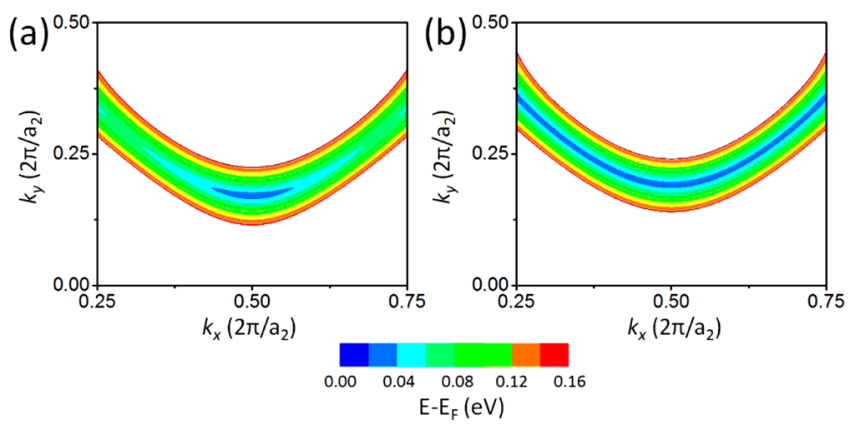

Figure 4. (Color online) (a) 2D constant-energy contour of band structure calculated from the TB model for 4,12,4-graphyne, (b) similar fitting results as that in (a) but only considering the nearestneighbor hopping.

interaction, whereas the nearest-neighbor interaction alone only produces a Dirac ring. Our findings enrich the Dirac physics founded in other 2D carbon allotropes and offer a new design mechanism for creating Dirac band by tuning the interaction range. We envision that this new type of Dirac crescent may be exploited in carbon-based electronic devices for manipulating anisotropic electron propagation.

\section{ASSOCIATED CONTENT}

\section{S Supporting Information}

Supporting Information includes the electronic structures of 4,12,2-graphyne, effective model fitting results for 4,12,2graphyne and 4,12,4-graphyne, anisotropic group velocity for 4,12,2- and 4,12,4-graphyne, tight-binding band structures for 4,12,2- and 4,12,4-graphyne and corresponding fitting parameters. The Supporting Information is available free of charge on the ACS Publications website at DOI: 10.1021/acs.jpclett.5b01337.

\section{AUTHOR INFORMATION}

\section{Corresponding Authors}

*E-mail: zhmwang@uestc.edu.cn.

*E-mail: sxdu@iphy.ac.cn.

*E-mail: fliu@eng.utah.edu.

Notes

The authors declare no competing financial interest.

\section{ACKNOWLEDGMENTS}

This work was partially supported by the Natural Science Foundation of China (Nos. 51325204, 61390501, and 51210003), the MOST 973 projects of China (No. 2011CB921702), the Chinese Academy of Sciences (CAS), Shanghai Supercomputer Center. Z.F.W. and F.L. acknowledge support from DOE-BES (Grant No. DE-FG02-04ER46148).

\section{REFERENCES}

(1) Castro Neto, A. H.; Peres, N. M. R.; Novoselov, K. S.; Geim, A. K. Rev. Mod. Phys. 2012, 81, 109.

(2) Novoselov, K. S.; Geim, A. K.; Morozov, S. V.; Jiang, D.; Katsnelson, M. I.; Grigorieva, I. V.; Dubonos, S. V.; Firsov, A. A. Nature 2005, 438, 197.

(3) Geim, A. K.; Novoselov, K. S. Nat. Mater. 2007, 6, 183.

(4) Ma, Y. D.; Dai, Y.; Huang, B. B. J. Phys. Chem. Lett. 2013, 4, 2471.

(5) Malko, D.; Neiss, C. F.; Viñes; Görling, A. Phys. Rev. Lett. 2012, 108, 086804.

(6) Cahangirov, S.; Topsakal, M.; Aktürk, E.; Şahin, H.; Ciraci, S. Phys. Rev. Lett. 2009, 102, 036804. 
(7) Zhou, X. F.; Dong, X.; Oganov, A. R.; Zhu, Q.; Tian, Y. J.; Wang, H. T. Phys. Rev. Lett. 2014, 112, 085502.

(8) Zhang, L. Z.; Wang, Z. F.; Du, S. X.; Gao, H.-J.; Liu, F. Phys. Rev. B: Condens. Matter Mater. Phys. 2014, 90, 161402.

(9) Wang, J. Y.; Deng, S. B.; Liu, Z. F.; Liu, Z. R. National Science Review 2015, 2, 22.

(10) Wang, Z. F.; Liu, Z.; Liu, F. Nat. Commun. 2013, 4, 1471.

(11) Zhou, M.; Ming, W. M.; Liu, Z.; Wang, Z. F.; Li, P.; Liu, F. Proc. Natl. Acad. Sci. U. S. A. 2014, 111, 14378.

(12) Zhou, M.; Liu, Z.; Ming, W. M.; Wang, Z. F.; Liu, F. Phys. Rev. Lett. 2014, 113, 236802.

(13) Park, C. H.; Yang, L.; Son, Y. W.; Cohen, M. L.; Louie, S. G. Nat. Phys. 2008, 4, 213.

(14) Wang, Z. F.; Liu, F. ACS Nano 2010, 4, 2459.

(15) Pardo, V.; Pickett, W. E. Phys. Rev. Lett. 2009, 102, 166803.

(16) Banerjee, S.; Singh, R. R. P.; Pardo, V.; Pickett, W. E. Phys. Rev. Lett. 2009, 103, 016402.

(17) Xu, L. C.; Wang, R. Z.; Miao, M. S.; Wei, X. L. Y. P.; Chen; Yan, H.; Lau, W. M.; Liu, L. M.; Ma, Y. M. Nanoscale 2014, 6, 1113.

(18) Huang, H. Q.; Duan, W. H.; Liu, Z. R. New J. Phys. 2013, 15, 023004.

(19) Enyashin, A. N.; Ivanovskii, A. L. Phys. Status Solidi B 2011, 248, 1879.

(20) Li, G. X.; Li, Y. L.; Liu, H. B.; Guo, Y. B.; Lia, Y. J.; Zhua, D. B. Chem. Commun. 2010, 46, 3256.

(21) Baughman, R. H.; Eckhardt, H.; Kertesz, M. J. Chem. Phys. 1987, $87,6687$.

(22) Zhao, M. W.; Dong, W. Z.; Wang, A. Z. Sci. Rep. 2013, 3, 03532.

(23) Yang, D. Z.; Si, M. S.; Zhang, G. P.; Xue, D. S. Europhys. Lett. 2014, 107, 20003.

(24) Kim, B. G.; Choi, H. J. Phys. Rev. B: Condens. Matter Mater. Phys. 2012, 86, 115435.

(25) Wang, J. Y.; Huang, H. Q.; Duan, W. H.; Liu, Z. R. J. Chem. Phys. 2013, 139, 184701.

(26) Liu, Z. R.; Wang, J. Y.; Li, J. L. Phys. Chem. Chem. Phys. 2013, $15,18855$.

(27) Vanderbilt, D. Phys. Rev. B: Condens. Matter Mater. Phys. 1990, $41,7892$.

(28) Kresse, G.; Furthmuller, J. Phys. Rev. B: Condens. Matter Mater. Phys. 1996, 54, 11169.

(29) Blöchl, P. E. Phys. Rev. B: Condens. Matter Mater. Phys. 1994, 50, 17953.

(30) Ceperley, D. M.; Alder, B. J. Phys. Rev. Lett. 1980, 45, 566.

(31) Perdew, J. P.; Zunger, A. Phys. Rev. B: Condens. Matter Mater. Phys. 1981, 23, 5048.

(32) Parlinski, K.; Li, Z. Q.; Kawazoe, Y. Phys. Rev. Lett. 1997, 78, 4063.

(33) Togo, A.; Oba, F.; Tanaka, I. Phys. Rev. B: Condens. Matter Mater. Phys. 2006, 78, 134106.

(34) Diederich, F. Nature 1994, 369, 199.

(35) Bunz, U. H. F.; Rubin, Y.; Tobe, Y. Chem. Soc. Rev. 1999, 28, 107.

(36) Sakamoto, J.; van Heijst, J.; Lukin, O.; Schlüter, A. D. Angew. Chem., Int. Ed. 2009, 48, 1030.

(37) Montambaux, G.; Piéchon, F.; Fuchs, J.-N.; Goerbig, M. O. Phys. Rev. B: Condens. Matter Mater. Phys. 2009, 80, 153412. 